\title{
A comparative longitudinal study of bovine trypanosomiasis in tsetse-free and tsetse- infested zones of the Amhara Region, Northwest Ethiopia
}

\begin{abstract}
A study was conducted to determine the incidence of trypanosome infections in cattle in tsetse-free and tsetse-infested zones of the Amhara Region of northwest Ethiopia. A total of six sentinel herds were established and the cattle observed during a period of 8 consecutive months. The prevalence of seropositive cattle was high in both the tsetse-free and tsetseinfested zones. The average monthly incidence of trypanosome infection, determined using molecular diagnostic tools, was $20.9 \%$ and $25.7 \%$ in the tsetse-free and the tsetse-infested zones, respectively. In the tsetse-free, Trypanosoma vivax was responsible for $90.9 \%$ of the cattle trypanosome infections. In the tsetse-infested zone, Trypanosoma congolense and T. vivax contributed almost equally to the trypanosome infections in cattle. Trypanosome infection, regardless of species, resulted in anaemia as evidenced by a significant decrease in the packed cell volume of the infected animal. The outcome of this longitudinal study suggests that control of trypanosomiasis in the Amhara Region cannot be achieved by tsetse control alone. Supplemental measures to include drug therapy and biting fly control are discussed.
\end{abstract}

Keyword: Trypanosoma, Transmission, Ethiopia 1 